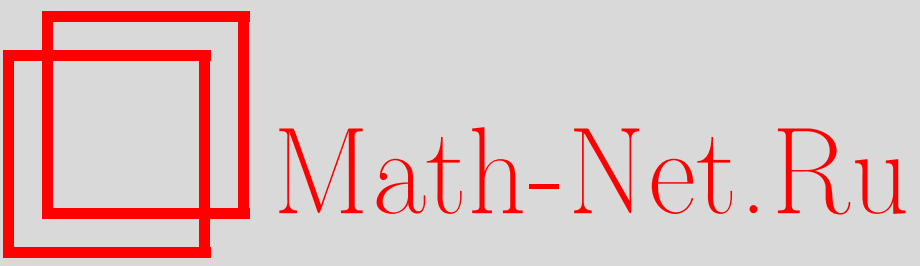

В. Бальзер, Проблема приведения Биркгофа, УМН, 2004, том 59, выпуск 6, 4154

DOI: https://doi.org/10.4213/rm794

Использование Общероссийского математического портала Math-Net.Ru подразумевает, что вы прочитали и согласны с пользовательским соглашением http: //www . mathnet.ru/rus/agreement

Параметры загрузки:

IP: 54.81 .137 .203

26 апреля 2023 г., 14:36:01 


\title{
ПРОБЛЕМА ПРИВЕДЕНИЯ БИРКГОФА
}

\author{
В. БАЛЬЗЕР
}

\begin{abstract}
Проблема приведения Биркгофа состоит в отыскании аналитического или мероморфного преобразования, которое приводит линейную мероморфную систему обькновенных дифференциальных уравнений к виду, в котором коэффициенты являются полиномами. В этой статье дан обзор существующих резултатов, а также представлено несколько новых результатов. В частности, показано, что каждая неприводимая система с неразветвленной формалной фундаментальной матрицей решений может быть преобразована к биркгофовой стандартной форме, т.е. к системе минималшного ранга Пуанкаре с нормализованными собственными значениями в нуле.

Библиография: 19 названий.
\end{abstract}

\section{СОДЕРЖАНИЕ}

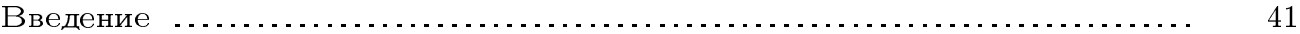

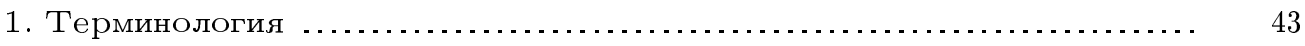

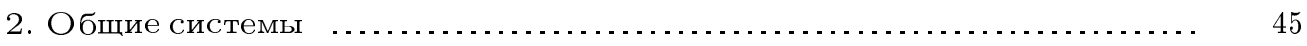

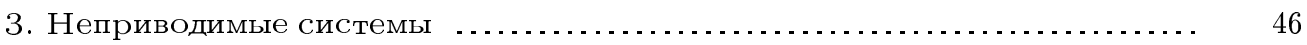

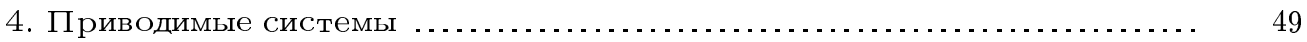

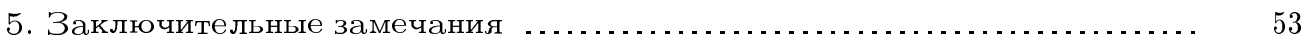

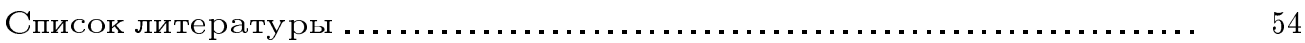

\section{Введение}

Эта статья посвяшена локальной теории линейных однородных систем обыкновенных дифференциальных уравнений, коэффициенты которых, по предположению, являются голоморфньми функциями вне круга радиуса $\rho \geqslant 0$ с центром в нуле и имеют, самое большее, полюс в бесконечности. Такие системы будут записываться следуюшим образом:

$$
z x^{\prime}=A(z) x, \quad A(z)=z^{r} \sum_{n=0}^{\infty} A_{n} z^{-n}, \quad|z|>\rho, \quad A_{0} \neq 0,
$$

где $r$ - положительное целое число, которое мы будем назьвать рангом Пуанкаре или просто рангом системы (1). Коэффициенты $A_{n}$, а следовательно, и $A(z)$ являются матрицами фиксированного размера $\nu \times \nu$ с натуральным числом $\nu \geqslant 2$, и разложение $A(z)$ предполагается сходяшимся при $|z|>\rho$. При $r \geqslant 1$ эта система имеет 
особенности второго рода в $z=\infty$, кроме того, мы будем дополнительно предполагать, что бесконечность является иррегулярной особенностью. Это означает, что решения растут экспоненциально, когда $z \rightarrow \infty$ в некоторых секторах конечного раствора. Последнее предположение делается, поскольку для регулярных особых точек задачи, которые будут здесь исследоваться, становятся тривиальными.

Изучение систем такого вида имеет долгую, однако все еше не завершенную историю. Здесь мы еще раз обратимся к одному вопросу, которьй был поставлен Джс. Биркгофом [8] в 1913 г. Он предложил, что для того, чтобы понять поведение решений системы (1) при $z \rightarrow \infty$, надо сначала найти линейное преобразование $x=T(z) y$ с обратимой матрищей $T(z)$, которая имеет "хорошее поведение" вблизи $\infty$ и преобразует систему (1) к системе

$$
z y^{\prime}=B(z) y, \quad B(z)=T^{-1}(z)\left[A(z) T(z)-z T^{\prime}(z)\right]
$$

c “как можно более простой” новой матрицей коэффициентов $B(z)$. Затем, когда это сделано, надо изучить решения упрошенной системы (2) и из их поведения в $z=\infty$ и природы матрицы $T(z)$ вывести заключение о решениях первоначальной системы (1). Сам Биркгоф рассмотрел матрищы $T(z)$, которые позже были названы аналитическими преобразованиями ${ }^{1}$. Множество аналитических преобразований образует группу относительно умножения матриц и, следовательно, индуцирует отношение эквивалентности на множестве всех систем вида (1). В статье, упомянутой выше, Биркгоф (ошибочно) утверждал, что в каждом классе эквивалентности найдется представитель класса (2), который имеет полиномиальную матрицу коэффициентов $B(z)$. Такая полиномиальная система, кроме бесконечности, имеет еще только одну особенность в нуле, которая есть особенность первого рода. Следовательно, ее решения, без монодромийных членов, являются целыми функциями конечного экспоненциального порядка, разложения которых в степенные ряды, по крайней мере теоретически, могут быть рекурсивно вычислены по коэффициентам разложения полиномиальной матрицы $B(z)$. Хотя эта полиномиальная система неоднозначно определяется по первоначальной системе (1), тем не менее ее можно рассматривать как (локальную) нормальную форму системы (1). Биркгоф показал в [8], что такие нормальные формы сушествуют при дополнительном предположении диагонализируемости матрищы монодромии системы (1), однако был убежден, что тот же результат верен в общем случае. Тем не менее, в 1953 г. Гантмахер [12] и в 1959 г. Мазани [16] независимо привели примеры систем вида (1), в наименьшей нетривиальной размерности $\nu=2$, для которых не существует аналитического преобразования к биркгофовой стандартной форме. Поскольку эти контрпримеры были треугольньми и, следовательно, приводимылми системами, это ведет к вопросу: каждая ли неприводимая система аналитически эквивалентна системе с полиномиальной матрицей коэффициентов? Эта аналитическая проблема приведения Биркгофа была решена положительно, сначала для размерности $\nu=2$ Д. Лутием, А. Пейеримховым и В.Б. Юркатом в [15], затем для $\nu=3$ В. Бальзером в [2] и, наконец, для всех размерностей $A$. А. Болибрухом в [10], [11].

\footnotetext{
${ }^{1}$ Точные определения этого и нескольких других терминов, использованных здесь, приведены в начале следующего раздела. В действительности, Биркгоф в дальнейшем ограничил используемые преобразования требованием $T(\infty)=I$, что, однако, не имеет значения для наших целей.
} 
Используя мероморфные преобразования вместо аналитических, соответствуюший вариант результата Биркгофа показьвает, что мы можем преобразовать каждую систему к полиномиальной системе, но с бо́льшим рангом Пуанкаре. Поскольку этот ранг играет решающую роль в определении поведения решений в бесконечности, такого возрастания ранга необходимо, по возможности, избегать. Поэтому встал вопрос: каждая ли система (1) может быть приведена с помощью мероморфного преобразования к полиномиальной системе (2) с тем же, или даже меньшим, рангом $r$ ? Эту задачу в дальнейшем будем назьвать мероморфной проблемой приведения Биркгофа. Для размерности $\nu=2$ (соответственно $\nu=3$ ) Лути, Пейеримхов u Юркат в [15] (соответственно Бальзер в [1]) показали, что ответ в мероморфной проблеме приведения Биркгофа положительный. Для произвольной размерности, но при дополнительном предположении о ведущей матрище $A_{0}$ системы (1) (она должна иметь различные собственные значения) $X$. Л. Турритин [19] также получил положительньй ответ, а в [4] можно найти другие достаточные условия, при которых известно, что ответ положительный. Однако в общем случае эта проблема остается открытой до сих пор.

Личные замечания автора. Я был потрясен, усльшав о безвременной кончине Андрея Болибруха в 2003 г. Хотя мы были друзьями в течение многих лет, я узнал о том, как серьезно болен Андрей, лишш за год до его смерти. Когда мы встречались в Москве в августе 2002 г. и когда я позже звонил ему в госпиталь, он был полон оптимизма, строил планы нашей будушей работы над проблемой приведения Биркгофа и говорил, что получил недавно новые результаты в исследовании мероморфного варианта проблемы, в частности, положительньй ответ в размерности четыре. Теперь мы, может быть, никогда не узнаем об этом результате, и это потеря для математического сообшества. Для меня несравненно большая потеря - утрата друга. Но мне даже трудно представить, сколь велика эта потеря для его семьи.

\section{1. Терминология}

В этой статье мы будем пользоваться следующей терминологией, которая, с одной стороны, является стандартной, а с другой, уже была успешно использована Бальзером, Лутием и Юркатом [5]-[7] при создании теории инвариантов как аналитических, так и мероморфных преобразований систем вида (1).

(a) Матричный степенной ряд вида $\widehat{T}(z)=\sum_{k=0}^{\infty} T_{k} z^{-k}$ (по переменной $z^{-1}$ ), чей свободный член $T_{0}$ является обратимой матрицей, будет рассматриваться в качестве формального аналитического преобразования. Если степенной ряд сходится для достаточно больших $|z|$, мы будем писать $T(z)$ вместо $\widehat{T}(z)$ и говорить аналитическое преобразование, опуская прилагательное формальное. Заметим, что было бы более точно назвать $T(z)$ матричей преобразования, резервируя термин преобразования для линейной замены переменной $x=T(z) y$ для системы (1), но для простоты языка мы не будем здесь делать этого различия.

(b) Скажем, что матрица $T(z)$ есть мероморфное преобразование, если ее элементы являются голоморфньми функциями вне некоторого круга с центром в нуле, имеют самое большее полюс в $z=\infty$ и, дополнительно, детерминант $\operatorname{det} T(z)$ не равен тождественно нулю. Эти условия обеспечивают, что $T^{-1}(z)$ также является мероморфным преобразованием. Мероморфные преобразования всегда могут быть разложены в ряд Лорана $T(z)=\sum_{k=k_{0}}^{\infty} T_{k} z^{-k}$ для подходящего целого числа $k_{0}$, и этот ряд схо- 
дится для достаточно больших значений $|z|$. Подобно ситуации аналитических преобразований, мы будем назьвать любой формальныи ия рядорана $\widehat{T}(z)=\sum_{k=k_{0}}^{\infty} T_{k} z^{-k}$ формальнылм мероморфным преобразованием при условии, что его детерминант не является нулевым рядом.

(c) Каждую фундаментальную матрицу решений $X(z)$ системы (1) можно голоморфно продолжить вдоль любой положительно ориентированной окружности радиуса, большего чем $\rho$, и результируюшая фундаментальная матрища решений будет, в обшем случае, отличаться от $X(z)$; поэтому обозначим ее $X\left(z e^{2 \pi i}\right)$. Тогда существует постоянная обратимая матрица (обозначим ее $e^{2 \pi i M}$ ), для которой выполняется равенство

$$
X\left(z e^{2 \pi i}\right)=X(z) e^{2 \pi i M} .
$$

Это эквивалентно равенству $X(z)=S(z) e^{2 \pi i M}$, где $S(z)$ - голоморфная однозначная матрица при $|z|>\rho$. Мы будем ссылаться на матрицу $M$ как на матрицу монодромии. Напомним, что всегда можно так выбрать матрицу $M$, что ее собственные значения будут нормализованы, т.е. их вещественные части будут содержаться в полуинтервале $[0,1)$. Если последнее условие выполнено, то матрица $M$ однозначно сопоставляется фундаментальной матрице решений $X(z)$ и, в частности, собственные значения матрищы $M$ являются инвариантами мероморфных преобразований.

(d) Систему (2), связанную с системой (1) аналитическим (соответственно мероморфным) преобразованием, будем называть аналитически (соответственно мероморфно) әквивалентной системе (1). Заметим, что в случае аналитической эквивалентности обе системы имеют один и тот же ранг Пуанкаре, в то время как в общем случае мероморфного преобразования их ранги могут отличаться. Однако заметим, что по предположению система (1) имеет иррегулярную особенность в бесконечности, которая таковой остается при любом мероморфном преобразовании системы. Следовательно, в любом классе эквивалентности относительно мероморфных преобразований существуют системы с минимальным рангом Пуанкаре, и мы будем говорить о таких системах как о системах минимального ранга. Заметим, что этот минимальньй ранг является формальным мероморфным инвариантом в смысле работы [5].

(е) Каждая система (1) допускает формальную фундаментальную матрицу решений вида

$$
\widehat{X}(z)=\widehat{F}(t) z^{L} e^{Q(t)},
$$

где $t=z^{1 / p}$ для некоторого натурального числа $p, \widehat{F}(t)$ - формальное мероморфное преобразование, матрица $Q(t)$ является диагональной матрицей с полиномами от $t$ на диагонали, нормализованной условием $Q(0)=0$, а матрица $L$ является постоянной матрицей с собственными значениями, нормализованными в смысле, указанном выше. Если сушествует формальная фундаментальная матрища решений, для которой можно выбрать $p=1$, то мы будем назьвать систему (1) формально неразветвленной. В этом случае мы получаем равенство (в формальном смысле) $\widehat{X}\left(z e^{2 \pi i}\right)=\widehat{X}(z) e^{2 \pi i L}$, и потому матрица $L$ будет назьваться матрицей формальной монодромии.

(f) Систему (2) будем называть в этой статье биркгофовой стандартной формой системы (1) при условии, что обе системы являются (мероморфно) эквивалентными и $B(z)$ является полиномиальной матрицей от $z$ степени не выше $r$. В случае, когда все собственные значения постоянной матрицы $B(0)$ имеют вещественные части в полуинтервале $[0,1)$, мы будем говорить, что система (2) имеет нормализованные собственные значения. 
(g) Систему (1) будем назьвать приводимой, если она мероморфно эквивалентна системе (2) с блочно-нижнетреугольной матрицей коэффициентов $B(z)$. Если это не так, мы будем говорить о неприводимой системе.

Для приводимых систем неважно, используются ли мероморфные или аналитические преобразования для получения блочно-треугольной формы. Относительно этого и многих других результатов о приводимых системах для сравнения см. работу [7].

\section{2. Общие системы}

Как упомянуто во введении, следующая теорема содержится в [8]. Для полноты мы приведем здесь ее доказательство.

Теорема 1 (Дж. Биркгоф, 1913). Кажсдая система (1) аналитически эквивалентна системе с особенностями только в бесконечности и в нуле, причем нуль будет регулярной особой точкой. В случае, когда система (1) имеет фундаментальную матрицу решений с диагональной матрицей монодромии, система (1) даже аналитически әквивалентна системе в биркгофовой стандартной форме.

ДокАЗАТЕльство. Сформулированная теорема является немедленным следствием общего результата о факторизации голоморфных матриц, которьй независимо был получен Д. Гильбертом [13], Й. Племелем [17], Дж. Биркгофом [9], а легче отыскиваемое его изложение находится в книге Й. Сибуйи [18].

Каждая матричная функиия $S(z)$ размера $\nu \times \nu$, голоморфная при $|z|>\rho$, детерминант которой не обращается в нуль при этих значениях $z$, может быть разложена в следующее произведение:

$$
S(z)=T(z) E(z) z^{K}
$$

где $T(z)$ - аналитическое преобразование, $E(z)$ - челая голоморфная матричная функция, детерминант которой не равен нулю для любых $z \in \mathbb{C}, u K=$ $\operatorname{diag}\left[k_{1}, \ldots, k_{\nu}\right]-$ челочисленная диагональная матрица.

Чтобы применить этот результат, возьмем фундаментальную матрицу решений $X(z)$ с матрицей монодромии $M$, так что $S(z)=X(z) z^{-M}$ является однозначной матричной функцией при $|z|>\rho$. Тогда детерминант $\operatorname{det} S(z)$ не равен нулю для этих $z$. Для $T(z)$ из разложения (4) преобразование $x=T(z) y$ переводит систему (1) в систему (2), имеюшую фундаментальную матрицу решений $Y(z)=E(z) z^{K}$. Таким образом, равенство

$$
B(z)=z Y^{\prime}(z) Y^{-1}(z)=\left[z E^{\prime}+E(z)\left\{K+z^{K} M z^{-K}\right\}\right] E^{-1}(z)
$$

показывает, что матрица $B(z)$ голоморфна и однозначна в комплексной плоскости $\mathbb{C}$, исключая нуль. Более того, мы видим из разложения $Y(z)$, что нуль является регулярной особой точкой системы $(2)$, и матрица $B(z)$ будет полиномиальной тогда и только тогда, когда матрица $z^{K} M z^{-K}$ будет полиномиальной. Это, конечно, имеет место, если $M$ - диагональная матрица.

Из доказательства теоремы 1 мы заключаем, что в случае диагональной матрищы монодромии $M$ имеет место равенство $B(0)=E(0)(M+K) E^{-1}(0)$. Следовательно, собственные значения матрищы $B(0)$ сравнимы по модулю 1 с собственными значениями матрицы монодромии $M$ (справедливость этого также можно увидеть, используя 
теорию особенностей первого рода ${ }^{2}$ ). Для многих целей желательно было бы иметь стандартную биркгофову форму с нормализованными собственными значениями, однако вопрос, можно ли добиться сказанного без увеличения ее ранга, остается открытым.

Тем не менее для формально неразветвленных систем с диагональной матрицей формальной монодромии мы докажем существование такой биркгофовой стандартной формы. Этот результат был анонсирован без доказательства В. Б. Юркатом [14; с. 178] и содержит в себе теорему Г. Л. Турритина [19] для систем, у которых коэффициент $A_{0}$ имеет различные собственные значения.

Теорема 2. Каждая система (1), которая формально неразветвлена и имеет диагональную матрицу формальной монодромии, мероморфно эквивалентна биркгофовой стандартной форме (2) минимального ранга и с нормализованными собственными значениями.

ДокаЗАТЕльство. Согласно [1; теорема 1], каждая система (1) мероморфно эквивалентна системе (2) с полиномиальной матрищей коэффициентов $B(z)$, которая имеет нормализованные собственные значения (однако в общем случае с рангом Пуанкаре, большим чем $r$ ), и формальной фундаментальной матрицей решений $\widehat{X}(z)=$ $\widehat{F}(z) z^{K} G(z)$. Здесь $\widehat{F}(z)$ - формальное аналитическое преобразование, $K$ - диагональная целочисленная матрица и $G(z)$ - формальный мероморфный инвариант системы (1) в смысле работы [5]. Для формально неразветвленных систем имеем $G(z)=$ $z^{L} e^{Q(z)}$, где $Q(z)$ - диагональная матрица полиномов от $z$, а постоянная матрица $L$ коммутирует с $Q(z)$. Следовательно, ранг Пуанкаре системы (2) равен рангу системы $z\left(z^{K} G(z)\right)^{\prime} G^{-1}(z) z^{-K}=K+z^{K} L z^{-K}+z Q^{\prime}(z)$. Известно, что минимальньй ранг системы будет равен степени полиномиальной матрицы $z Q(z)$ и будет равен минимальному рангу системы (2), если матрицы $L$ и $z^{K}$ коммутируют, а это, конечно, так, если $L-$ диагональная матрица.

Читатель мог заметить некоторую аналогию доказательств двух теорем, представленных вьше. Первое использует преобразования, которые не изменяют природу особенности системы (1) в бесконечности, но имеют отношение к виду матрищы монодромии, которая тесно связана с поведением решений полиномиальной системы вблизи нуля. Второе доказательство использует матрицу формальной монодромии (формальных) решений вблизи бесконечности, и даже когда эта матрица $L$ не диагональная, можно надеяться продвинуться в доказательстве того же самого результата, используя дополнительные мероморфные преобразования, которые помогут так изменить матрицу $K$, чтобы получить коммутирование матриц $L$ и $z^{K}$, в то же время оставляя особенность в нуле неизменной. Эта двойственность двух особых точек будет играть ключевую роль также и в последующем изложении.

\section{3. Неприводимые системы}

Как было сказано выше, $A$. A . Болибрух в работах [10], [11] показал, что любая неприводимая система аналитически эквивалентна системе в биркгофовой стандартной форме. Мы приведем здесь эту теорему и изложим главные идеи ее доказательства,

\footnotetext{
${ }^{2}$ Речь идет о теории особых точек с нулевым рангом Пуанкаре или, в другой терминологии, о фуксовых особых точках. (Прим. ред. перевода.)
} 
для того чтобы показать, что получаемая стандартная форма в общем случае не имеет нормализованных собственных значений.

ТеОрема 3 (А.А. Болибрух, 1994). Каждая неприводимая система аналитически әквивалентна такой жее системе в биркгофовой стандартной форме.

ДоКАЗАТЕЛЬСтво. Выберем фундаментальную матрицу решений $X(z)=S(z) z^{J}$ системы (1) с матрицей монодромии $J$, имеющей верхнетреугольную жорданову форму. Пусть $D=\operatorname{diag}\left[d_{1}, \ldots, d_{\nu}\right]$ - диагональная матрища с целочисленными элементами на диагонали, удовлетворяющими условию $d_{j}-d_{j+1} \geqslant r(\nu-1)$. Тогда произведение $S(z) z^{-D}$ можно представить в виде

$$
S(z) z^{-D}=T(z) z^{K} E(z)
$$

с множителями $T(z), z^{K}$ и $E(z)$ того же типа, что и в (4); однако эти два представления различны, поскольку последние два множителя не коммутируют. Без потери обшности можно считать, что диагональные элементы $k_{j}$ матрицы $K$ уменьшаются. Доказательство факта, что такая факторизация, вместо (4), возможна, можно найти, например, в работе [3; с. 191]. Для матрицы $\widetilde{B}(z)=T^{-1}(z)\left[A(z) T(z)-z T^{\prime}(z)\right]$ система $z \widetilde{y}^{\prime}=\widetilde{B}(z) \widetilde{y}$ имеет ранг $r$ и обладает фундаментальной матрицей решений вида $\widetilde{Y}(z)=z^{K} E(z) z^{D} z^{J}$. Это показьвает, что выполняется равенство

$$
z^{-K} \widetilde{B}(z) z^{K}=K+\left[z E^{\prime}(z)+E(z)\left(D+z^{D} J z^{-D}\right)\right] E^{-1}(z) .
$$

Так как матрица $J$ верхнетреугольная и диагональные элементы $d_{j}$ убьвают, мы видим, что правая часть равенства голоморфна в нуле. Если сушествует некоторое $j$, для которого вьполняется неравенство $k_{j}-k_{j+1}>r$, то тот факт, что система $z \widetilde{y}^{\prime}=$ $\widetilde{B}(z) \widetilde{y}$ имеет ранг Пуанкаре $r$, влечет, что матрица $\widetilde{B}(z)$ будет блочно-треугольной. Это, однако, противоречит неприводимости системы (1). Следовательно, вьполняются неравенства $0 \leqslant k_{j}-k_{j+1} \leqslant r$ для всех $1 \leqslant j \leqslant \nu-1$. Используя упражнение из [3; с. 192], мы заключаем, что имеют место разложения $\widetilde{Y}(z)=\widetilde{T}(z) Y(z)$ с аналитическим преобразованием $\widetilde{T}(z)$ и $Y(z)=\widetilde{E}^{ \pm}(z) z^{\widetilde{K}+D} z^{J}$, где $E^{ \pm}(z)$ являются целыми голоморфными функциями, а диагональная матрица $\widetilde{K}$ равна матрице $K$ с точностью до некоторой перестановки диагональных элементов. Следовательно, мы можем заключить, что $\left|\widetilde{k}_{j}-\widetilde{k}_{j+1}\right| \leqslant(\nu-1) r$ для $j=1, \ldots, \nu-1$. Исходя из нашего выбора матрищы $D$, мы найдем, что диагональные элементы $\widetilde{K}+D$ являются убьваюшими, так что матрица $B(z)=z Y^{\prime}(z) Y^{-1}(z)$ будет голоморфной в нуле, т.е., в действительности, является полиномиальной матрицей. Поскольку система с такой матрицей аналитически эквивалентна системе (1), доказательство завершено.

ЗАмЕчАНИЕ 1. Для последующего важно заметить, что биркгофова стандартная форма, полученная в доказательстве вьше, имеет матрицу коэффициентов $B(z)$ со значением в нуле $B(0)$, которое имеет собственные значения, равные диагональным элементам матрицы $\widetilde{K}+D+\Lambda$, где $\Lambda$ - диагональная часть матрицы монодромии $J$. Поскольку доказательство требует выбора диагональных элементов матрицы $D$ с достаточно большой разностью, мы видим, что в общем случае нельзя ожидать, что стандартная форма будет иметь нормализованные собственные значения. Однако матрицу $D$ можно выбрать так, что некоторые собственные значения сдвигаются на целое 
число сколь угодно далеко влево на комплексной плоскости, в то время как другие движутся вправо (заметим, что, как следует из рассмотрения следа системы, аналитические преобразования не изменяют суммы собственных значений матрицы $B(0))$. В частности, выбирая различные матрицы $D$, можно показать, что существует бесконечно много различных биркгофовых стандартных форм для неприводимых систем (1).

Когда используются мероморфные преобразования вместо аналитических, теорема Болибруха может быть применена, чтобы получить биркгофову стандартную форму с минимальным рангом. А именно, для заданной неприводимой системы мы можем сначала использовать мероморфные преобразования, понижаюшие ее ранг настолько, насколько это возможно, а затем аналитическими преобразованиями привести к стандартной биркгофовой форме, чей ранг теперь минимальньй. Однако из того, что сказано в замечании 1 , ясно, что эта стандартная форма будет в общем случае иметь ненормализованные собственные значения. Поскольку это свойство очень важно для вычисления фундаментальной матрицы решений (около нуля), мы покажем теперь, что для формально неразветвленных неприводимых систем стандартная форма, на самом деле, может быть получена с помошю мероморфных преобразований. Заметим, что доказательство следующей теоремы имеет много аналогий с доказательством результата Болибруха, а также теоремы 2.

ТЕОРема 4. Каждая формально неразветвленная неприводимая система (1) мероморфно әквивалентна такой же системе в биркгофовой стандартной форме, имеющей минимальный ранг и нормализованные собственные значения.

ДокАЗАТЕльство. Согласно [1; теорема 1], система (1) мероморфно эквивалентна системе (2) с полиномиальной матрицей коэффициентов $B(z)$, которая имеет нормализованные собственные значения (однако, вообще говоря, имеет ранг Пуанкаре, больший чем $r$ ) и формальную фундаментальную матрицу решений вида $\widehat{X}(z)=\widehat{F}(z) z^{K} G(z)$ с формально аналитическим преобразованием $\widehat{F}(z)$, диагональной целочисленной матрищей $K=\operatorname{diag}\left[k_{1}, \ldots, k_{\nu}\right]$ и формальным мероморфным инвариантом $G(z)$ системы (1) в смысле [5]. Для простоты мы будем полагать, что такова уже исходная система (1). Согласно нашим предположениям, формальньй мероморфный инвариант имеет вид

$$
G(z)=z^{J} e^{Q(z)}
$$

с диагональной матрицей $Q(z)$ из полиномов (от $z$ ) и матрицей формальной монодромии $J$, которая коммутирует с $Q(z)$ и которая, как здесь мы предполагаем, имеет нижнетреугольную жорданову форму. Пусть диагональная матрица $D=\operatorname{diag}\left[d_{1}, \ldots, d_{\nu}\right]$ имеет целочисленные диагональные элементы, удовлетворяющие условию $d_{j}-d_{j+1} \geqslant$ $r(\nu-1)$; сопоставление с доказательством леммы 2 из работы [1] показьвает, что можно разложить

$$
\widehat{F}(z) z^{K-D}=\widetilde{P}(z) z^{\widetilde{K}} \widehat{T}(z)
$$

где $\widetilde{P}(z)$ - полиномиальная матрица, детерминант которой никогда не обращается в нуль, $\widetilde{K}$ - диагональная матрица из целых чисел, которые убывают, а $\widehat{T}(z)$ - формальное аналитическое преобразование. Мероморфное преобразование $x=\widetilde{P}(z) \widetilde{y}$ приводит к системе с полиномиальной матрицей коэффищиентов $\widetilde{B}(z)$, имеюшей формальную фундаментальную матрицу решений $z^{\widetilde{K}} \widehat{T}(z) z^{D} z^{J} e^{Q(z)}$. Это, вместе с нижней 
треугольностью матрицы $J$ и фактом, что диагональные элементы матрищы $D$ убывают, может быть использовано для проверки того, что матрица $z^{-\widetilde{K}} \widetilde{B}(z) z^{\widetilde{K}}$ имеет ранг Пуанкаре, равньй $r$. Следовательно, если неравенство $\widetilde{k}_{j}-k_{j+1}>r$ вьполняется для какого-либо $j$, то матрица $\widetilde{B}(z)$ - блочно-треугольная, а это противоречит неприводимости системы (1). Таким образом, $0 \leqslant \widetilde{k}_{j}-\widetilde{k}_{j+1} \leqslant r$ для каждого $j$. Как и в доказательстве леммы 2 из работы [1], мы видим, что имеет место равенство

$$
\widehat{F}(z) z^{\check{K}-D}=P(z) \check{F}(z) z^{\check{K}}
$$

где $P(z)$ - другая полиномиальная матрица, детерминант которой нигде не обращается в нуль, $\check{K}$ - диагональная целочисленная матрица, которая отличается от матрицы $\widetilde{K}$ некоторой перестановкой диагональных элементов, и $\check{F}(z)$ является формальным аналитическим преобразованием. Мероморфное преобразование $x=P(z) y$ приводит к системе (2) с полиномиальной матрицей коэффициентов $B(z)$, имеющей формальную фундаментальную матрищу решений вида $\check{F}(z) z^{D+K} z^{J} e^{Q(z)}$. При подходящем выборе матрицы $D$ диагональные элементы матрицы $D+\check{K}$ убывают, поэтому ранг системы (2) равен рангу системы

$$
\begin{aligned}
C(z) & \left.=z\left(z^{D+\check{K}} z^{J} e^{Q(z)}\right)^{\prime}\left(z^{D+\check{K}} z^{J} e^{Q(z)}\right)\right)^{-1} \\
& =D+\check{K}+z^{D+\check{K}} z^{J} z^{-D-\check{K}}+z Q^{\prime}(z),
\end{aligned}
$$

которая имеет минимальньй ранг. Поскольку преобразование $x=P(z) y$ не изменяет собственных значений постоянного члена матрицы коэффициентов, доказательство завершено.

ЗАмЕчАнИЕ 2. Заметим, что последняя теорема показьвает, что существует биркгофова стандартная форма с формальной фундаментальной матрищей решений $\widehat{X}(z)=\check{F}(z) z^{D+\check{K}} z^{J} e^{Q(z)}$. Поскольку $\check{F}(z)$ является формальнылм аналитическим преобразованием, мы можем считать (5) матрицей коэффищиентов формальной стандартной формы системы (1). Отметим, что доказательство требует выбора матришы $D$, чьи диагональные элементы имеют достаточно большие разности, и это приводит к формальной стандартной форме $C(z)$ с собственными значениями матришы $C(0)$, отличающимися друг от друга на достаточно большое целое число (см. замечание 1).

\section{4. Приводимые системы}

Приводимые системы, по определению, эквивалентны системам с (нижнетреугольной) блочной матрищей коэффициентов. Предположим дополнительно, что диагональные блоки все являются неприводимыми, в противном случае мы можем получить эквивалентную матрицу с более тонкой блочно-треугольной структурой. Чтобы найти фундаментальную матрищу решений такой системы, требуется отыскание решений (меньших) систем, соответствуюших диагональным блокам, с последуюшим выгислением нескольких интегралов. Грубо говоря, нетривиальная часть этого вычисления сосредоточена только на неприводимых системах. По этой причине можно было бы подумать, что вопрос о биркгофоовом приведении является второстепенным вопросом, 
однако досадно, что до сих пор мы не знаем, может ли это быть сделано (с помощью мероморфного преобразования) или существуют какие-либо контрпримеры. Здесь мы изучим несколько достаточных условий, при которых биркгофова стандартная форма может быть получена.

Для простоты обозначений мы ограничимся блочно-нижнетреугольными системами вида

$$
z x^{\prime}=A(z) x, \quad A(z)=\left[\begin{array}{cc}
A_{1}(z) & O \\
A_{21}(z) & A_{2}(z)
\end{array}\right],
$$

с квадратными диагональными блоками, возможно различных размеров $s_{1}$ и $s_{2}$, и $O$ обозначает блок с тождественно нулевьми элементами. Заметим, что каждая приводимая система по определению мероморфно эквивалентна указанной системе, так что без ограничения обшности будем предполагать выписанную выше форму системы, когда будут обсуждаться приводимые системы. Главное дополнительное предположение состоит в том, что диагональные блоки (6) являются полиномиальными $n o$ z. Иногда мы будем рассматривать случаи, когда система $z x_{2}^{\prime}=A_{2}(z) x_{2}$, соответствующая второму диагональному блоку, является неприводимой. Однако, чтобы включить в рассмотрение системы с более тонкой блочно-нижнетреугольной структурой (с более чем двумя диагональными блоками), мы не будем предполагать неприводимости системы $z x_{1}^{\prime}=A_{1}(z) x_{1}$. Налагая различные ограничения на два диагональных блока в процессе наших рассмотрений, мы дадим достаточные условия для сушествования аналитических или мероморфных преобразований очень специального вида

$$
x=T(z) y, \quad T(z)=\left[\begin{array}{cc}
I & O \\
T_{21}(z) & I
\end{array}\right],
$$

которые приводят систему к биркгофовой стандартной форме (2). Эта система затем также приводится к блочному виду, подобному (6), с диагональными блоками $B_{j}(z)=$ $A_{j}(z)$ и

$$
B_{21}(z)=A_{21}(z)+A_{2}(z) T_{21}(z)-T_{21}(z) A_{1}(z)-z T_{21}^{\prime}(z) .
$$

Однако заметим, что вовсе неясно, будут ли также всегда иметь блочно-нижнетреугольную структуру биркгофовы стандартные формы для блочно-нижнетреугольных систем, или, если это так, то будут ли две блочные структуры совпадать.

4.1. Аналитические преобразования. Доказательство следуюшего результата можно найти в [4].

Теорема 5. Пусть матрица $A(z)$ будет такой же, как в системе (6), и с такими полиномиальными диагональными блоками, что для каждого челого числа $k \geqslant 1$ матрицы $A_{1}(0)$ и $A_{2}(0)+k I$ не имеют одинаковых собственных значений. Тогда существует такое аналитическое преобразование вида (7), которое преобразует систему (6) к биркгофовой стандартной форме.

Можно добиться вьполнения условий, наложенных в последней теореме на собственные значения, применяя теорему 4 к каждому диагональному блоку в максимально возможной тонкой блочной структуре, в которой диагональные блоки неприводимы, или используя так назьваемые срезающие преобразования вида $T(z)=\operatorname{diag}\left[z^{k} I_{s_{1}}, I_{s_{2}}\right]$ с подходящим целым $k$. Однако, поскольку обе возможности используют мероморфные преобразования, мы столкнемся с ситуациями, в которых 
ранг Пуанкаре системы (6) определяется внедиагональньм блоком. В таких случаях можно было бы использовать другие срезаюшие преобразования, чтобы понизить ранг, но они могут привести к системам, в которых предположение на собственные значения не выполняется. По этой причине сформулированная вьше теорема не является достаточной для решения мероморфной версии проблемы приведения Биркгофа.

Следуюшая теорема немного более общая, чем теорема 2 из работы [4], но может быть доказана при помощи почти тех же самых рассуждений.

Теорема 6. Пусть матрица $A(z)$ - такая жее, как в системе (6), и имеет полиномиальные диагональные блоки, а система $z x_{2}^{\prime}=A_{2}(z) x_{2}$ является неприводимой. Более того, предположим, что матрииа $A_{2}(0)$ имеет, по крайней мере, одно собственное значение $\mu$, которое не равно по модулю иелого числа ни одному из собственных значений матрицы $A_{1}(0)$. Тогда существует аналитическое преобразование, которое приводит систему (6) в блочно-нижнетреугольную биркгофову стандартную форму.

ДокАЗАТЕЛьСТво. Главная идея доказательства состоит в таком применении теоремы 3 и соответственно замечания 1 ко второму диагональному блоку, что собственное значение $\mu$ заменяется на $\mu-k$ с подходяшим большим $k$, в то время как другие собственные значения сдвигаются вправо в комплексной плоскости, так что все предположения на собственные значения из теоремы 5 вьполняются. Подробности могут быть найдены в доказательстве теоремы 2 из [4].

4.2. Мероморфные преобразования. В этом разделе мы еще раз более подробно рассмотрим систему (6), делая следуюшие дополнительные предположения.

(i) Система (6) - формально неразветвленная система.

(ii) Меньшие подсистемы

$$
z x_{j}^{\prime}=A_{j}(z) x_{j}, \quad 1 \leqslant j \leqslant 2,
$$

соответствуюшие диагональным блокам (6), имеют нормализованные собственные значения и приведены к биркгофовым стандартньм формам с минимальными рангами (которым разрешается отличаться друг от друга и быть меньше, чем ранг $r$ системы (6)).

(iii) Системы (9) имеют формальные фундаментальные матрицы решений вида

$$
\widehat{X}_{j}(z)=\widehat{F}_{j}(z) z^{K_{j}} z^{L_{j}} z^{Q_{j}(z)},
$$

где $\widehat{F}_{j}(z)$ являются формальными аналитическими преобразованиями, в то время как $K_{j}$ и $Q_{j}(z)$ являются диагональными матрицами с целочисленными и полиномиальными элементами соответственно, а $L_{j}$ являются постоянными матрицами, коммутируюшими с соответствующими матрицами $Q_{j}(z)$.

Заметим, что в случае системы (6), в которой обе подсистемы (9) являются неприводимьми, мы можем применить теорему 4 к каждому диагональному блоку, что приведет к новой системе с диагональными блоками, которые удовлетворяют предположению (ii), сделанному выше. Более того, принимая во внимание само доказательство вышеназванной теоремы, можно также увидеть, что имеется возможность устроить так, чтобы предположение (iii) также имело силу. Следовательно, сушественньм 
в этом случае из наших предположений является предположение (i). Для более общих систем мы все еше можем полагать второй блок неприводимьм, и, таким образом, наши предположения, отличные от первого и касающиеся второго блока, не являются ограничительньми.

Для последующего важно иметь формальную фундаментальную матрицу решений системы (6), которая является блочно-нижнетреугольной. Существование такого решения следует из результатов работ [14], [5], и мы докажем, что можно выбрать фундаментальную матрицу решений следуюшего вида.

Лемма 1. При предположениях, сделанных выше, система (6) имеет формальную фундаментальную матрицу решений следующего вида:

$$
\widehat{X}(z)=\widehat{F}(z) z^{K} z^{L} z^{Q(z)} .
$$

Здесь $Q(z)=\operatorname{diag}\left[Q_{1}(z), Q_{2}(z)\right], K=\operatorname{diag}\left[K_{1}, K_{2}\right] u$

$$
\widehat{F}(z)=\left[\begin{array}{cc}
\widehat{F}_{1}(z) & 0 \\
\widehat{F}_{21}(z) & \widehat{F}_{2}(z)
\end{array}\right], \quad L=\left[\begin{array}{cc}
L_{1} & 0 \\
L_{21} & L_{2}
\end{array}\right],
$$

постоянная матрица $L_{21}$ удовлетворяет соотношению $Q_{2}(z) L_{21}=L_{21} Q_{1}(z)$, формальный ряд $\widehat{F}_{21}(z)$ и остальнье матричь такие же, как в (10).

ДокАЗАТЕльство. Заметим, что блочно-нижнетреугольная матрица $\widehat{X}(z)$, чьи диагональные блоки равны $\widehat{X}_{1}(z), \widehat{X}_{2}(z)$ (как в $(10)$ ), является формальной фундаментальной матрицей решений системы (6) тогда и только тогда, когда ее внедиагональньй блок $\widehat{X}_{21}(z)$ удовлетворяет неоднородному уравнению

$$
z \widehat{X}_{21}^{\prime}=A_{21}(z) \widehat{X}_{1}+A_{22}(z) \widehat{X}_{21}
$$

Это уравнение, в свою очередь, эквивалентно равенству $\widehat{X}_{21}(z)=\widehat{X}_{2}(z) \widehat{U}(z)$, где $\widehat{U}(z)$ - формальная первообразная выражения $z^{-1} \widehat{X}_{2}^{-1}(z) A_{21} \widehat{X}_{1}(z)$. Согласно $[14 ; \S 13]$, такие первообразные сушествуют в классе формальных логарифмически-экспоненциальных выражений (и даже единственны, если избегать необязательных констант интегрирования). Более того, можно найти, что $\widehat{X}_{21}(z)=\widehat{\Psi}_{21}(z) e^{Q_{1}(z)}$ с формальным логарифммическим выражением $\widehat{\Psi}_{21}(z)$. Теория формальных решений влечет тогда существование такой блочно-треугольной матрищы $L$, коммутирующей с $Q(z)$, что $\widehat{X}\left(z e^{2 \pi i}\right)=\widehat{X}(z) e^{2 \pi i L}$, и это показывает, что выражение $\left.\widehat{X}(z) e^{-Q(z)} z^{-L}\right)$ является (формально) однозначньм формальным логарифмическим выражением, т.е. формальным рядом Лорана. Используя эти факты, доказательство может быть завершено.

С помощью этой леммы мы теперь докажем следующий результат, которьй содержит теорему 3 из [4].

ТеОрема 7. При предположениях, сделанньх выше, пусть $L_{21}$ будет как в (11), и положим, что матрица $z^{K_{2}} L_{21} z^{-K_{1}}$ имеет ранг не больше, чем максимум рангов матрич $A_{1}(z)$ и $A_{2}(z)$. Тогда существует мероморфное преобразование вида (7), превращающее систему (6) в биркгофову стандартную форму с минимальныцм рангом и нормализованным.ми собственнылми значениями. 
ДокАЗАТЕЛьство. Преобразование $T(z)$ будет строиться в два этапа. Возьмем формальную фундаментальную матрицу решений $\widehat{X}(z)$ как в лемме 1, и пусть $T_{21}(z)$ будет длинный, но конечный кусок формального ряда Лорана $\widehat{F}_{21}(z) \widehat{F}_{1}(z)^{-1}$. Соответствующее преобразование (7) преврашает систему (6) в систему того же вида, но с другим внедиагональным блоком, которая имеет формальную фундаментальную матрицу решений того же типа, что в лемме 1 , но с заменой $\widehat{F}_{21}(z)$ на $\widehat{F}_{21}(z)-T_{21}(z) \widehat{F}_{1}(z)$. Согласно выбору блока $T_{21}(z)$ (и тому факту, что $\widehat{F}_{1}(z)$ - формально аналитический ряд), новьй блок $\widehat{F}_{21}(z)$ не содержит никаких членов, соответствующих положительным степеням $z$. Другими словами, мы можем считать, что имеем дело с системой (6), обладаюшей формальной фундаментальной матрицей решений как в лемме 1 , но с формально аналитическим $\widehat{F}(z)$. В этом случае мы можем показать, используя коммутативность матрищ $L$ и $Q(z)$, что ранг Пуанкаре системы (6) равен рангу Пуанкаре системы

$$
z\left(z^{K} z^{L} e^{Q(z)}\right)^{\prime}\left(z^{K} z^{L} e^{Q(z)}\right)^{-1}=K+z Q^{\prime}(z)+z^{K} L z^{-K} .
$$

При сделанных предположениях этот ранг является минимальным и из теоремы 5 следует существование аналитического преобразования (7) к биркгофовой стандартной форме. Такое преобразование не изменяет ни ранга, ни собственных значений первоначальной системы; это завершает доказательство.

\section{5. Заключительные замечания}

Подчеркнем, что мероморфную версию проблемы приведения Биркгофа можно подразделить на две части: первая часть состоит в отыскании мероморфного преобразования, приводящего к системе с полиномиальной матрицей коэффициентов; существование такого преобразования следует из обших результатов о факторизации голоморфных матриц, которые цитировались в теореме 1 . Этот результат можно рассматривать как трансцендентную часть проблемы, поскольку существование такого преобразования гарантируется, но его никогда не удается вычислить, за исключением тривиальных случаев. Вторая часть проблемы имеет дело с отысканием другого преобразования, которое преврашает полиномиальную систему в другую полиномиальную систему меньшего ранга Пуанкаре, имеющую (если это возможно) нормализованные собственные значения. Это преобразование обязательно имеет лорановское разложение, которое состоит из конечного числа членов, и, следовательно, его отыскание является чисто алгебраической задачей. Удивительно, что сушествование такого преобразования все еше не доказано во всех случаях, и поэтому эта часть проблемы должна рассматриваться как более трудная! Также отметим, что для полиномиальной системы матрица монодромии и/или экспоненщиальная часть, а также формальная монодромия формальной фундаментальной матрицы решений могут быть вычислены по системе, и, таким образом, по крайней мере теоретически, можно проверить предположения, сделанные во всех предыдуших теоремах, - исключая неприводимость, которая использует информацию о матрицах Стокса системы.

Все результаты, представленные здесь, получены с использованием преобразований, которые изменяют некоторые существенные данные одной из двух особенностей полиномиальной системы, но оставляют их неизменными в другой особенности. Этот подход хорошо работает для неприводимых систем, однако, по-видимому, недостаточен для других систем. Причина этого, по мнению автора, кроется в том, что для при- 
водимых систем характеры двух особенностей взаимосвязаны. Так что в этом случае приходится использовать преобразования, которые одновременно действуют на обе особые точки. По-видимому, можно найти стратегию, в которой применяются очень простые преобразования и которая, шаг за шагом, улучшает структуру особенностей и неизбежно приводит к системе минимального ранга в биркгофовой стандартной форме или к неулучшаемой системе; такая стратегия являлась бы контрпримером к проблеме мероморфного приведения. К сожалению, никто в настояшее время, по-видимому, не может сделать это.

\section{СПИСОК ЛИТЕРАТУРЫ}

[1] W. Balser. Meromorphic transformation to Birkhoff standard form in dimension three // J. Fac. Sci. Univ. Tokyo Sect. IA Math. 1989. V. 36. № 2. P. 233-246.

[2] W. Balser. Analytic transformation to Birkhoff standard form in dimension three // Funkcial. Ekvac. 1990. V. 33. № 1. P. 59-67.

[3] W. Balser. Formal Power Series and Linear Systems of Meromorphic Ordinary Differential Equations. New York: Springer-Verlag, 2000.

[4] W. Balser, A. A. Bolibruch. Transformations of reducible equations to Birkhoff standard form // Ulmer Seminare - Funktionalanalysis und Differentialgleichungen, University Ulm, 1997; http://www.mathematik.uni-ulm.de/m5/balser/papers.html.

[5] W. Balser, W.B. Jurkat, D. A. Lutz. A general theory of invariants for meromorphic differential equations. I. Formal invariants // Funkcial. Ekvac. 1979. V. 22. № 2. P. 197-221.

[6] W. Balser, W. B. Jurkat, D. A. Lutz. A general theory of invariants for meromorphic differential equations. II. Proper invariants // Funkcial. Ekvac. 1979. V. 22. № 3. P. 257-283.

[7] W. Balser, W. B. Jurkat, D. A. Lutz. Invariants for reducible systems of meromorphic differential equations // Proc. Edinburgh Math. Soc. (2). 1980. V. 23. № 2. P. 163-186.

[8] G.D. Birkhoff. Equivalent singular points of differential equations // Math. Ann. 1913. V. 74. P. 134-139

[9] G.D. Birkhoff. A theorem on matrics of analytic functions // Math. Ann. 1913. V. 74. P. $122-133$.

[10] А. А. Болибрух. Об аналитическом преобразовании к биркгофовой стандартной форме // Труды МИАН. 1994. Т. 203. С. 29-35.

[11] А. А. Болибрух. Об аналитическом преобразовании к биркгофовой стандартной форме // Докл. РАН. 1994. Т. 334. № 5. С. 553-555.

[12] Ф.Р. Гантмахер. Теория матриц. М.: Гостехиздат, 1953.

[13] D. Hilbert. Grundzüge einer allgemeinen Theorie der linearen Integralgleihungen (Dritte Mitt.) // Nachr. Ges. Wiss. Göttingen. 1905. P. 307-338.

[14] W. B. Jurkat. Meromorphe Differentialgleichungen. Berlin: Springer-Verlag, 1978. (Lecture Notes in Math. V. 637.)

[15] W. B. Jurkat, D. A. Lutz, A. Peyerimhoff. Birkhoff invariants and effective calculations for meromorphic linear differential equations // J. Math. Anal. Appl. 1976. V. 53. № 2. P. $438-470$

[16] P. Masani. On a result of G. D. Birkhoff on linear differential systems // Proc. Amer. Math. Soc. 1959. V. 10. P. 696-698.

[17] J. Plemelj. Riemannsche Funktionenscharen mit gegebener Monodromiegruppe // Monatsh. Math. Phys. 1908. V. 19. P. 211-246.

[18] Y. Sibuya. Linear Differential Equations in the Complex Domain: Problems of Analytic Continuation. Providence, RI: Amer. Math. Soc., 1990. (Transl. Math. Monogr. V. 82.)

[19] H. L. Turrittin. Reduction of ordinary differential equations to the Birkhoff canonical form // Trans. Amer. Math. Soc. 1963. V. 107. P. 485-507.

Universität Ulm, Germany

Поступила в редакцию

E-mail: balser@mathematik.uni-ulm.de

27.07 .2004 